\title{
(6) Safety and effectiveness of influenza vaccines
}

\author{
Unexpected safety signals and new data challenge our assumptions
}

\section{Heath A Kelly \\ MBBS, MPH Head, Epidemiology Unit \\ Victorian Infectious Diseases Reference Laboratory, Peter Doherty Institute, Melbourne, VIC. \\ heath.kelly@mh.org.au}

doi: 10.5694/mjal4.01281

Research p 596

$\mathrm{n}$ this issue of the Journal, Wood and colleagues report the results of a study showing that the inactivated influenza vaccines currently licensed for use in children in Australia are reassuringly safe. ${ }^{1}$ Study nurses at multiple sites around Australia telephoned parents to collect data on fever and other adverse events after vaccination. In the sample of 893 children aged 6 months to $<10$ years, the estimated risk of fever within 72 hours of influenza vaccine receipt was about $6 \%$ after the first or second dose. The risk of fever was higher if another vaccine was given at the same time. Only one febrile convulsion was reported, in a child with a known seizure disorder.

Until a few years ago, the safety of influenza vaccines was not a concern in Australia because no significant safety problem with their administration to adults or children had been reported through passive surveillance in more than 50 years. All this changed in 2010 when vaccines manufactured by bioCSL were shown to cause fever at 5-10 times previously accepted risk levels and febrile convulsions at 200 times higher levels. ${ }^{2}$ These bioCSL vaccines must no longer be used in children aged under 5 years and should only be used with caution in children aged 5 to 9 years.

\section{Selected global studies monitoring annual influenza vaccine effectiveness}

\section{Study}

\section{Southern hemisphere}

Influenza Complications Alert Network (FluCAN) $)^{12}$

Victorian sentinel general practice surveillance scheme ${ }^{9}$

Western Australian Influenza Vaccine Effectiveness (WAIVE) Study ${ }^{10}$

Southern Hemisphere Influenza Vaccine Effectiveness, Research and Surveillance (SHIVERS) study ${ }^{18}$

Northern hemisphere

Canadian sentinel surveillance network ${ }^{19}$

Influenza Monitoring Vaccine

Effectiveness (I-MOVE) network ${ }^{20}$

US Influenza Vaccine Effectiveness (Flu VE) Network ${ }^{21}$

Primary Health Care Sentinel Network and Network for Influenza Surveillance in Hospitals of Navarre ${ }^{22}$

\section{Design and setting}

Pooled sentinel hospitals in Australia

Sentinel general practices in Victoria

Emergency department presentations and hospital inpatients at Princess Margaret Hospital for Children, Perth

Population-based study in sentinel general practices and hospitals in Auckland, New Zealand Pooled sentinel general practices in Canada Pooled sentinel general practices in 6-8 European countries

Pooled community-based sentinel research sites in the United States

Population-based study in sentinel general practices and hospitals in the Navarre region of Spain
After extensive investigations over more than 3 years, bioCSL attributed the increased fever risk of the vaccine to a combination of its manufacturing process and strain changes in some years. ${ }^{3}$ This is a potential concern because influenza vaccines often require strain changes and a future strain change might also be associated with unexpected safety problems. Because only a few months elapse between strain selection and vaccine availability, there is no time for annual safety or efficacy trials of influenza vaccines. Efficacy is assumed from immunogenicity assays, based on older studies reporting the relationship between haemagglutination antibody titres and protection of $50 \%$ of vaccine recipients. ${ }^{4}$ Post-infection ferret antisera and limited human data are used for specific strain selection. ${ }^{5}$ Safety is assumed from the absence of prior reported adverse events. However, the experience of 2010 suggests the assumption that past safety predicts present safety might not be valid.

Assumptions about efficacy have also been questioned recently. As late as 2012, the World Health Organization asserted that influenza vaccines were $70 \%-90 \%$ effective. ${ }^{6}$ While this may be true for live attenuated influenza vaccines given to children, it does not appear to be true for inactivated vaccines for any age group. Two recent metaanalyses report point estimates of efficacy for inactivated influenza vaccines against laboratory-confirmed influenza in community-based trials as $59 \%$ and $52 \%-65 \%$, with the latter range depending on the degree of match between the circulating and vaccine strains. In Australia, observational studies that collected data over 4 or 5 years found point estimates of vaccine effectiveness of $62 \%$ in Victorian adults aged 20-64 years ${ }^{9}$ and $65 \%$ in Western Australian children aged 6 months to 5 years.$^{10}$ However, vaccine effectiveness did not reach $50 \%$ in the Victorian community in $2012^{11}$ or in Australian patients hospitalised with influenza in the 2 years before that. ${ }^{12}$ Protection of older adults, who are targeted for vaccination, was particularly poor against influenza A(H3N2) in 2012. ${ }^{11}$

With the exceptions of an adjuvanted monovalent influenza vaccine manufactured by GlaxoSmithKline, which was used in Europe during the 2009 influenza pandemic and increased the risk of narcolepsy in children and young adults, ${ }^{13}$ and the bioCSL vaccine given to children (not just in 2010), ${ }^{2,14}$ most commentators accept that influenza vaccines are safe, based on the administration of many millions of doses over many years. However, 
unexpected safety signals show that such expectations will not always be met. ${ }^{2,13}$

Influenza vaccines with changed strains are considered to be variations of an existing vaccine, rather than new vaccines. As such, efficacy and safety studies are not required; nor are they practical in the time available. However, in the light of unexpected safety signals and sometimes low estimates of effectiveness, annual monitoring of the safety and effectiveness of influenza vaccines now seems like good public policy. It is therefore encouraging to see the safety study in this issue of the Journal. ${ }^{1}$ Timely safety monitoring should become a funded routine component of the influenza vaccine program in Australia. Effectiveness monitoring may not be as timely as the new mobile phone and internet-based strategies for safety monitoring already being piloted in Australia, ${ }^{15-17}$ but regular effectiveness monitoring should also become routine. Such monitoring is evolving throughout the world, including in Australia (Box), with many study sites able to derive timely interim effectiveness estimates using the case test-negative design. ${ }^{19-23}$ Monitoring of both safety and effectiveness is needed to assess existing vaccines and vaccines that may be introduced in the near future, such as live attenuated influenza vaccines for children.

Competing interests: No relevant disclosures.

Provenance: Commissioned; externally peer reviewed.

1 Wood NJ, Blyth CC, Willis GA, et al. The safety of seasonal influenza vaccines in Australian children in 2013. Med J Aust 2014; 201: 596-600.

2 Armstrong PK, Dowse GK, Effler PV, et al. Epidemiological study of severe febrile reactions in young children in Western Australia caused by a 2010 trivalent inactivated influenza vaccine. BMJ Open 2011; 1: e000016. do: 10.1136/bmjopen-2010-000016.

3 Rockman S, Becher D, Dyson A, et al. Role of viral RNA and lipid in the adverse events associated with the 2010 Southern Hemisphere trivalent influenza vaccine. Vaccine 2014; 32: 3869-3876.

4 Kelly $\mathrm{H}$, Barr I. Large trials confirm immunogenicity of $\mathrm{H} 1 \mathrm{~N} 1$ vaccines. Lancet 2010; 375: 6-9.

5 Klimov Al, Garten R, Russell C, et al. WHO recommendations for the viruses to be used in the 2012 Southern Hemisphere influenza vaccine: epidemiology, antigenic and genetic characteristics of influenza $A(\mathrm{HINI})$ pdm09, $A(H 3 N 2)$ and B influenza viruses collected from February to September 2011. Vaccine 2012; 30: 6461-6471.

6 Vaccines against influenza WHO position paper - November 2012. Wkly Epidemiol Rec 2012; 87: 461-476.

7 Osterholm MT, Kelley NS, Sommer A, Belongia EA. Efficacy and effectiveness of influenza vaccines: a systematic review and meta-analysis. Lancet Infect Dis 2012: 12: 36-44.
8 Tricco AC, Chit A, Soobiah C, et al. Comparing influenza vaccine efficacy against mismatched and matched strains: a systematic review and metaanalysis. BMC Med 2013, 11: 153. doi: 10.1186/1741-7015-11-153.

9 Kelly HA, Sullivan SG, Grant KA, Fielding JE. Moderate influenza vaccine effectiveness with variable effectiveness by match between circulating and vaccine strains in Australian adults aged 20-64 years, 2007-2011. Influenza Other Respir Viruses 2013; 7: 729-737.

10 Blyth CC, Jacoby P, Effler PV, et al. Effectiveness of trivalent flu vaccine in healthy young children. Pediatrics 2014; 133: el218-el225. doi: 10.1542/ peds.2013-3707.

11 Sullivan SG, Komadina N, Grant K, et al. Influenza vaccine effectiveness during the 2012 influenza season in Victoria, Australia: influences of waning immunity and vaccine match. J Med Virol 2014; 86: 1017-1025. doi: 10.1002/ jmv.23847.

12 Cheng AC, Holmes M, Irving LB, et al. Influenza vaccine effectiveness against hospitalisation with confirmed influenza in the 2010-11 seasons: a test-negative observational study. PLOS One 2013; 8: e68760. doi: 10.1371/ journal.pone.0068760.

13 Miller E, Andrews N, Stellitano L, et al. Risk of narcolepsy in children and young people receiving ASO3 adjuvanted pandemic A/HIN1 2009 influenza vaccine: retrospective analysis. BMJ 2013; 346: f794.

14 Lambert SB, Chuk LM, Nissen MD, et al. Safety and tolerability of a 2009 trivalent inactivated split-virion influenza vaccine in infants, children and adolescents. Influenza Other Respir Viruses 2013; 7: 676-685.

15 Regan AK, Blyth CC, Effler PV. Using SMS technology to verify the safety of seasonal trivalent influenza vaccine for pregnant women in real time [letter]. Med J Aust 2013; 199: 744-746.

16 Leeb A, Regan AK, Peters IJ, et al. Using automated text messages to monitor adverse events following immunisation in general practice. Med J Aust 2014; 200: 416-418.

17 Cashman P, Moberley S, Dalton C, et al. Vaxtracker: active on-line surveillance for adverse events following inactivated influenza vaccine in children. Vaccine 2014; 32: 5503-5508.

18 Turner N, Pierse N, Bisselo A, et al. Effectiveness of seasonal trivalent inactivated influenza vaccine in preventing influenza hospitalisations and primary care visits in Auckland, New Zealand, in 2013. Euro Surveill 2014; 19 : pii: 20884.

19 Skowronski D, Chambers C, Sabaiduc S, et al. Interim estimates of 2013/14 vaccine effectiveness against influenza $A(H 1 N 1) p d m 09$ from Canada's sentinel surveillance network, January 2014. Euro Surveill 2014; 19: pii: 20690.

20 Valenciano M, Kissling E; I-MOVE Case-Control Study Team. Early estimates of seasonal influenza vaccine effectiveness in Europe: results from the I-MOVE multicentre case-control study, 2012/13. Euro Surveill 2013; 18: pii: 20400.

21 Flannery B, Thaker SN, Clippard J, et al. Interim estimates of 2013-14 seasonal influenza vaccine effectiveness - United States, February 2014. Morb Mortal Wkly Rep 2014; 63: 137-142.

22 Castilla J, Martínez-Baz I, Navascués A, et al. Vaccine effectiveness in preventing laboratory-confirmed influenza in Navarre, Spain: 2013/14 midseason analysis. Euro Surveill 2014; 19: pii: 20700.

23 Foppa IM, Haber M, Ferdinands JM, Shay DK. The case test-negative design for studies of the effectiveness of influenza vaccine. Vaccine 2013; 31: 3104-3109. 